\title{
Assessing parasite clearance during uncomplicated Plasmodium falciparum infection treated with artesunate monotherapy in Suriname
}

This article was published in the following Dove Press journal:

Infection and Drug Resistance

24 November 2016

Number of times this article has been viewed

\section{Stephen GS Vreden' \\ Rakesh D Bansie ${ }^{2}$ \\ Jeetendra K Jitan ${ }^{3}$ \\ Malti R Adhin ${ }^{4}$}

'Foundation for Scientific Research Suriname (SWOS), ${ }^{2}$ Department of Internal Medicine, Academic Hospital Paramaribo, ${ }^{3}$ Department of Public Health, Ministry of Health, ${ }^{4}$ Department of Biochemistry, Anton de Kom University of Suriname, Paramaribo, Suriname
Correspondence: Stephen GS Vreden Foundation for Scientific Research Suriname (SWOS), Leysweg 49,

Paramaribo, Suriname

Tel +597880 715I

Email stephenvreden@yahoo.com
Background: Artemisinin resistance in Plasmodium falciparum is suspected when the day 3 parasitemia is $>10 \%$ when treated with artemisinin-based combination therapy or if $>10 \%$ of patients treated with artemisinin-based combination therapy or artesunate monotherapy harbored parasites with half-lives $\geq 5$ hours. Hence, a single-arm prospective efficacy trial was conducted in Suriname for uncomplicated P. falciparum infection treated with artesunate-based monotherapy for 3 days assessing day 3 parasitemia, treatment outcome after 28 days, and parasite half-life. Methods: The study was conducted in Paramaribo, the capital of Suriname, from July 2013 until July 2014. Patients with uncomplicated Plasmodium falciparum infection were included and received artesunate mono-therapy for three days. Day 3 parasitaemia, treatment outcome after 28 days and parasite half-life were determined. The latter was assessed with the parasite clearance estimator from the WorldWide Antimalarial Resistance Network (WWARN).

Results: Thirty-nine patients were included from July 2013 until July 2014. The day 3 parasitemia was $10 \%$. Eight patients $(20.5 \%)$ could be followed up until day 28 and showed adequate clinical and parasitological response. Parasite half-life could only be determined from ten data series $(25.7 \%)$. The median parasite half-life was 5.16 hours, and seven of these data series had a half-life $\geq 5$ hours, still comprising $17.9 \%$ of the total data series.

Conclusion: The low follow-up rate and the limited analyzable data series preclude clear conclusions about the efficacy of artesunate monotherapy in Suriname and the parasite half-life, respectively. The emergence of at least $17.9 \%$ of data series with a parasite half-life $\geq 5$ hours supports the possible presence of artemisinin resistance.

Keywords: malaria, falciparum, resistance, parasite half-life

\section{Background}

The emergence of artemisinin resistance in the Greater Mekong Subregion of Southeast Asia has raised concerns about the global threat of malaria and called for increased containment measures. The World Health Organization (WHO) recommends that therapeutic efficacy studies be performed once every 2 years in sentinel sites within each country for guiding antimalarial treatment policies. ${ }^{1}$ Artemisinin resistance is suspected when $\geq 10 \%$ of patients treated with artemisinin-based combination therapy (ACT) have positive parasitemia on day 3. Artemisinin resistance is confirmed in case of treatment failure with parasites persisting for 7 days in the blood stream or the presence of parasites at day 3 and recrudescence within 28/42 days after a treatment with oral artemisinin-based monotherapy. A treatment failure rate $>10 \%$ should prompt a change in the national antimalarial treatment policy. ${ }^{1}$ Since 2010 , the assessment of day 3 parasitemia during ACT treatment was used as a tool for screening artemisinin 
resistance and determination of the parasite half-life $(50 \%$ reduction in parasitemia during the linear phase of the log-parasitemia over time) was further incorporated in the assessment of artemisinin resistance. ${ }^{2,3}$ Artemisinin resistance could therefore also be suspected if $\geq 10 \%$ of patients treated with ACT or artesunate monotherapy harbored parasites with a half-life $\geq 5$ hours. ${ }^{4,5}$ The WHO criteria for artemisinin resistance have now been updated with the incorporation of K13-confirmed resistance mutations in which the presence of $\geq 5 \%$ of patients carrying $\mathrm{K} 13$ resistance-confirmed mutations can aid either alone or in combination with the aforementioned criteria in defining suspected or confirmed artemisinin resistance, respectively. ${ }^{1}$

The WorldWide Antimalarial Resistance Network (WWARN) established the parasite clearance estimator as a standardized method to assess the in vivo response to treatment with artemisinin derivatives. ${ }^{6}$

Owing to increased and structured efforts in the fight against malaria in Suriname, including the introduction of artemether-lumefantrine as the first-line treatment against Plasmodium falciparum in 2004, the incidence of malaria has decreased from 16,000 cases per year in 2001 to 374 cases in 2014 of which $P$. falciparum infections consisted of $43 \%$ of the cases. ${ }^{7}$ The majority of infections (>90\%) occur in border areas with artisanal and illegal gold mining, predominantly in miners from French Guiana. The efficacy of artemether-lumefantrine (Coartem) as the first-line treatment in Suriname was first evaluated in 2006, and an efficacy of $>95 \%$ was noted. ${ }^{8}$ In the following therapeutic efficacy study in 2011, a small sample size and a very high loss to followup rate, due to the significant decrease in malaria cases in Suriname and the high mobility of miners, respectively, prohibited conclusions for the conventional day 28 results. Only eleven of the 53 enrolled patients were available on day 28 , but all showed adequate clinical and parasitological response (ACPR). However, from the 48 patients available for day 3 sampling, a 31\% positive parasitemia on day 3 was observed, which was significantly higher compared to the $2 \%$ positive parasitemia on day 3 from the earlier study in $2006 .{ }^{9}$

To monitor emerging artemisinin resistance in Suriname, we conducted a single-arm prospective efficacy trial in patients with uncomplicated $P$. falciparum infection. In order to avoid confounding by partner drugs, the treatment used was oral artesunate-based monotherapy $4 \mathrm{mg} / \mathrm{kg}$ for 3 days with mefloquine and primaquine afterward. Determining the parasitemia every 8 hours for 3 days allowed assessment of artesunate efficacy through determination of the parasite clearance rate and the parasite half-life.

\section{Methods}

\section{Study site}

Suriname is situated in the northeastern region of South America and is bordered by French Guiana to the east, Brazil to the south, and Guyana to the west. Approximately $80 \%$ of its territory is covered by tropical rainforest where just $\sim 55,000$ people, of the $\sim 541,638$ inhabitants, live in small settlements throughout the Amazon rainforest. ${ }^{10}$ The population in the rain forest has increased due to the small-scale gold mining. ${ }^{11}$ It is in these mobile gold mining communities along the border with French Guiana where, hence, most of the transmission of malaria via Anopheles darlingi occurs. This study was conducted from July 2013 to July 2014 at the "Tourtonnelab", a malaria diagnostic and treatment facility in the north of Paramaribo, where most small-scale gold miners reside during their stay in the capital Paramaribo. This facility was specifically initiated by the Government of Suriname to facilitate early diagnosis and prompt treatment of this at-risk population. This study was approved by the national ethics committee (CMWO - Commissie voor Mensgebonden Wetenschappelijk Onderzoek) of the Ministry of Health in Suriname.

\section{Study design}

This study was a one-arm prospective evaluation of the clinical and parasitological responses to directly observed treatment with artesunate monotherapy $4 \mathrm{mg} / \mathrm{d}$ for 3 days for uncomplicated falciparum malaria. The patients were monitored for a minimum of 4 days, but if available were followed up to 28 days, to further assess the possible presence of artemisinin resistance as advised by the WHO guidelines and the parasite clearance rate.

\section{Study population}

The study population consisted of patients aged $\geq 2$ years with microscopically confirmed and uncomplicated $P$. falciparum malaria attending the clinic. Written informed consent was obtained from all adults. Consent from a parent or guardian was obtained for minors. To ensure minimal loss to followup, the patients were accommodated in a suitable hosting facility in the first 4 days.

Inclusion criteria for the study were: patients having a mono-infection with $P$. falciparum detected by microscopy, with 200-100,000 asexual parasites/ $\mu \mathrm{L}$; the presence of axillary or oral temperature $\geq 37.5^{\circ} \mathrm{C}$ or a history of fever during the past 24 hours; and the ability to swallow oral medication and to comply with the study protocol.

Patients were excluded from the study when signs of severe $P$. falciparum malaria according to the definitions of 
WHO, mixed or mono-infection with another Plasmodium species detected by microscopy, and severe malnutrition were present. Patients were further excluded if they had a febrile illness other than malaria or a chronic known underlying disorder or severe disease, regular medication that might interfere with antimalarial pharmacokinetics, a splenectomy, a history of hypersensitivity reactions or contraindications to any of the medicine(s) being evaluated, or used antimalarial drugs in the previous 48 hours. Women who were pregnant, lactating, or were unable or unwilling to take a pregnancy test or contraceptives (for women of childbearing age) were also excluded from the study.

The estimated prevalence of patients positive at day 3 after artesunate monotherapy $4 \mathrm{mg} / \mathrm{kg} / \mathrm{d}$ was $15 \%$ in Suriname. At a confidence level of $95 \%$ and a precision around the estimate of $15 \%$, a minimum of 40 patients had to be included. With a $10 \%$ increase to allow loss to follow-up and withdrawals during the 28-day follow-up period, 44 patients were targeted in the study.

\section{Initial assessment and follow-up}

Clinical and demographic assessments were performed on day 0. Urine pregnancy tests were performed for women. Standard clinical monitoring was further performed on days 1,2 , and 3 and, when possible, on days 7, 14, 21, and 28 . Microscopic examination was performed using Giemsastained thick and thin blood films on day 0 and also every 8 hours until 72 hours after the initial dose of artesunate and, when necessary, afterward daily until parasite clearance.

Patients were withdrawn from the study if they met the criteria for treatment failure, developed severe or complicated malaria, took additional self-administered antimalarial drugs or herbal remedies, had signs of persistent vomiting, or were lost to follow-up.

The numbers of parasites were counted per 200 leukocytes or per 500 leukocytes when $<10$ parasites per 200 leukocytes were present. The parasite density was expressed as parasites per microliter of blood, assuming a leukocyte count of $8,000 / \mu \mathrm{L}$. A blood slide was considered negative when the examination of 500 high-power fields revealed no asexual parasites. The presence of gametocytes did not contribute to the basic evaluation. Reading was done independently by two qualified microscopists, who were blinded to each other's reading, and parasite densities were afterward calculated as the average of the two counts. The slides were reevaluated after the study to assess the tenability and reproducibility by an external independent WHO-recognized senior expert microscopist.

\section{Therapy}

The patients received artesunate $4 \mathrm{mg} / \mathrm{kg} / \mathrm{d}$ (Guilin Pharmaceutical Company, Guilin, People's Republic of China) with tablets of $50 \mathrm{mg}$, rounded to the nearest quarter tablet once daily, for 3 days. After 72 hours of the initial dose of artesunate, they received mefloquine $250 \mathrm{mg}$ tablets, $25 \mathrm{mg} / \mathrm{kg}$ in one or two administrations, and primaquine $0.75 \mathrm{mg} / \mathrm{kg}$ in a single dose, as per national treatment protocol in order to terminate possible gametocytemia.

The administration of the drug and a 30-minute observation was done under the supervision of clinical staff. In cases of vomiting within this time, $100 \%$ of the original dose was given together with an antiemetic suppository. Additional antipyretics (in case of fever $>38^{\circ} \mathrm{C}$ ) were given when needed. If therapeutic failure, early treatment failure, and LTF ensued, the patient received artemether-lumefantrine.

In the presence of other malarial infections (Plasmodium vivax or Plasmodium malariae) or complications during treatment with artesunate, the excluded patients received alternative therapy according to the current national protocol and appropriate referral when needed.

\section{Outcome measures and data analysis}

Evaluable patients were classified as having early treatment failure, late clinical failure, and late parasitological failure or ACPR, according to the WHO guidelines. The percentages of patients with positive parasitemias on day 2 (PPP48) and day 3 (PPP72) were further assessed.

\section{Parasite clearance estimator}

The parasite clearance estimator established by WWARN was used to identify the lag phase, clean the data for outliers, and determine the best model (linear, quadratic, cuboidal) to fit the log-transformed parasite data and afterward calculate the following estimates: the parasite clearance rate; the parasite clearance time (the time required for complete clearance of the initial parasitemia, which is dependent upon this initial value); slope half-life, which is derived from the clearance rate constant and is independent of the initial parasitemia; and the time needed for parasitemia to fall by $50 \%, 90 \%, 95 \%$, and 99\% (PC50, PC90, PC95, and PC99, respectively). ${ }^{6}$ The WHO excel and SPSS Version 17 (SPSS Inc., Chicago, IL, USA) were used for data management and analysis.

\section{Results \\ Trial profile}

Forty-five patients with $P$. falciparum infection were evaluated from July 2013 to July 2014 from which 39 could be 
included in the study. The study was terminated despite not reaching the intended number of 40 due to the very low number of cases and the demand to limit the inclusion of patients to a maximum of 1 year. From the six patients who were excluded, two declined blood sampling, two were pregnant, one had a coinfection with $P$. vivax, and one used antimalarial medication prior to the study. The follow-up rates for days 3 and 28 were $97 \%$ and $21 \%$, respectively, for the 39 included patients.

\section{Demographic characteristics}

The study population consisted of $56 \%$ males and $44 \%$ females. The mean age and weight of the study population were $35 \pm 9.6$ years and $66.1 \pm 9.9 \mathrm{~kg}$, respectively. The youngest patient was aged 18 years and the oldest 53 years. All patients came from artisanal gold mining areas predominantly located in French Guiana (87\%), and only $13 \%$ originated from Suriname (6.5\%) and Guyana (6.5\%).

\section{Outcome measures}

There were no adverse events reported during the study. All eight patients who could be followed up until day 28 showed ACPR. There were no cases observed with early clinical failure, late clinical failure or late parasitological failure.

\section{Parasite clearance}

Parasite clearance data was only presented for the 30 data series, also examined by the external senior expert microscopist. Nine data series could not be determined at the time of external reevaluation due to mold contamination of slides after the long storage period and were therefore considered to be of compromised quality, as they might influence the reproducibility of the results, and were thus excluded from further analysis. The geometric mean for the number of asexual parasites on day 0 was $5,813 / \mu \mathrm{L}$ (range 1,201-84,743/ $\mu \mathrm{L})$. The number of patients with a positive parasitemia on day 2 was $18(62 \%)$. There were three patients with a positive parasitemia on day $3(10 \%)$. Comparative analysis of the field microscopy results with the results from the WHO expert showed identical results for the positive parasitemia on day $2(62 \%)$ and the positive (Table 1$)$.

For adequate estimation of the different parasite clearance kinetics parameters using the WWARN parasite clearance estimator, the initial parasitemia had to be $\geq 4,000$ asexual parasites $/ \mu \mathrm{L}$. Data from 20 patients $(66.7 \%)$ were therefore censored due to a parasite density $<4,000$ asexual parasites $/ \mu \mathrm{L}$.

Parasite clearance curves could be produced for ten patient data series (25.7\%). A lag phase was not detected in any of the
Table I Parasitological assessment

\begin{tabular}{|c|c|}
\hline \multicolumn{2}{|l|}{ Day 0 parasitemia ${ }^{a}$} \\
\hline Geometric mean $(95 \% \mathrm{Cl})$ & 5,813 \\
\hline Range & $\mathrm{I}, 20 \mathrm{I}-84,743$ \\
\hline IQR range & $2,858-10,369$ \\
\hline \multicolumn{2}{|c|}{ Patient with positive parasitemia ${ }^{\mathrm{a}}$ on day 2 and day 3} \\
\hline Positive parasitemia on day $2(\mathrm{~N}=29, \%)$ & $18 / 29(62)$ \\
\hline Positive parasitemia on day $3(\mathrm{~N}=30, \%)$ & $3 / 30(10)$ \\
\hline \multicolumn{2}{|l|}{ Parasite clearance rate constant (per hour) ${ }^{b}$} \\
\hline Median & 0.13 \\
\hline Range & $0.10-0.20$ \\
\hline IQR range & $0.13-0.14$ \\
\hline \multicolumn{2}{|l|}{ Slope half-life (hours) ${ }^{b}$} \\
\hline Median & 5.16 \\
\hline Range & $3.39-7.14$ \\
\hline IQR range & $4.84-5.46$ \\
\hline
\end{tabular}

Notes: ${ }^{a}$ Asexual parasites per microliter. ${ }^{b} n=10$.

Abbreviations: $\mathrm{Cl}$, confidence interval; IQR, interquartile range.

data series (Figure 1). The median clearance rate constant was 0.13 /hour (interquartile range [IQR] 0.01/hour). The median parasite half-life was 5.16 hours (IQR 0.62 hours). The distribution of the frequency of the parasite half-life is shown in Figure 2 and shows a unimodal distribution. Seven data series of patients had a parasite half-life $\geq 5$ hours (Table 2). These seven data series of patients represent $70 \%$ of the evaluated data series and still comprised $17.9 \%$ of the initial total of 39 patients included. The time to clear $50 \%, 90 \%, 95 \%$, and $99 \%$ of parasitemia is shown in Table 3 .

\section{Discussion}

\section{Treatment efficacy}

As was the case in earlier therapeutic efficacy trials for artemether-lumefantrine in 2005/2006 and 2011, the eight patients who could be followed up until day 28 (21\%) showed ACPR to artesunate monotherapy followed by mefloquine and primaquine. ${ }^{8,9}$ Several studies have illustrated the efficacy of artemisinin combination therapy or artesunate monotherapy despite prolonged clearance times. In Central Vietnam, where a median parasite half-life of 6.2 hours was found, the efficacy for dihydroartemisinin-piperaquine remained satisfactory. ${ }^{12}$ In a therapeutic efficacy study with artesunate monotherapy $4 \mathrm{mg} / \mathrm{kg} / \mathrm{d}$ for 7 days in Southern Myanmar in 2011, the authors found a half-life of 6.6 hours in the slower clearing half, but still achieved a $100 \%$ polymerase chain reactioncorrected cure rate. ${ }^{13}$ The administration of partner drugs after day 3, as was the case in our study, might contribute to the $100 \%$ efficacy. This premise is supported by the observed decrease in treatment failure rate in Pailin, Cambodia, an established region of artemisinin resistance, from $25 \%$ after the standard 3-day dihydroartemisinin-piperaquine regimen 

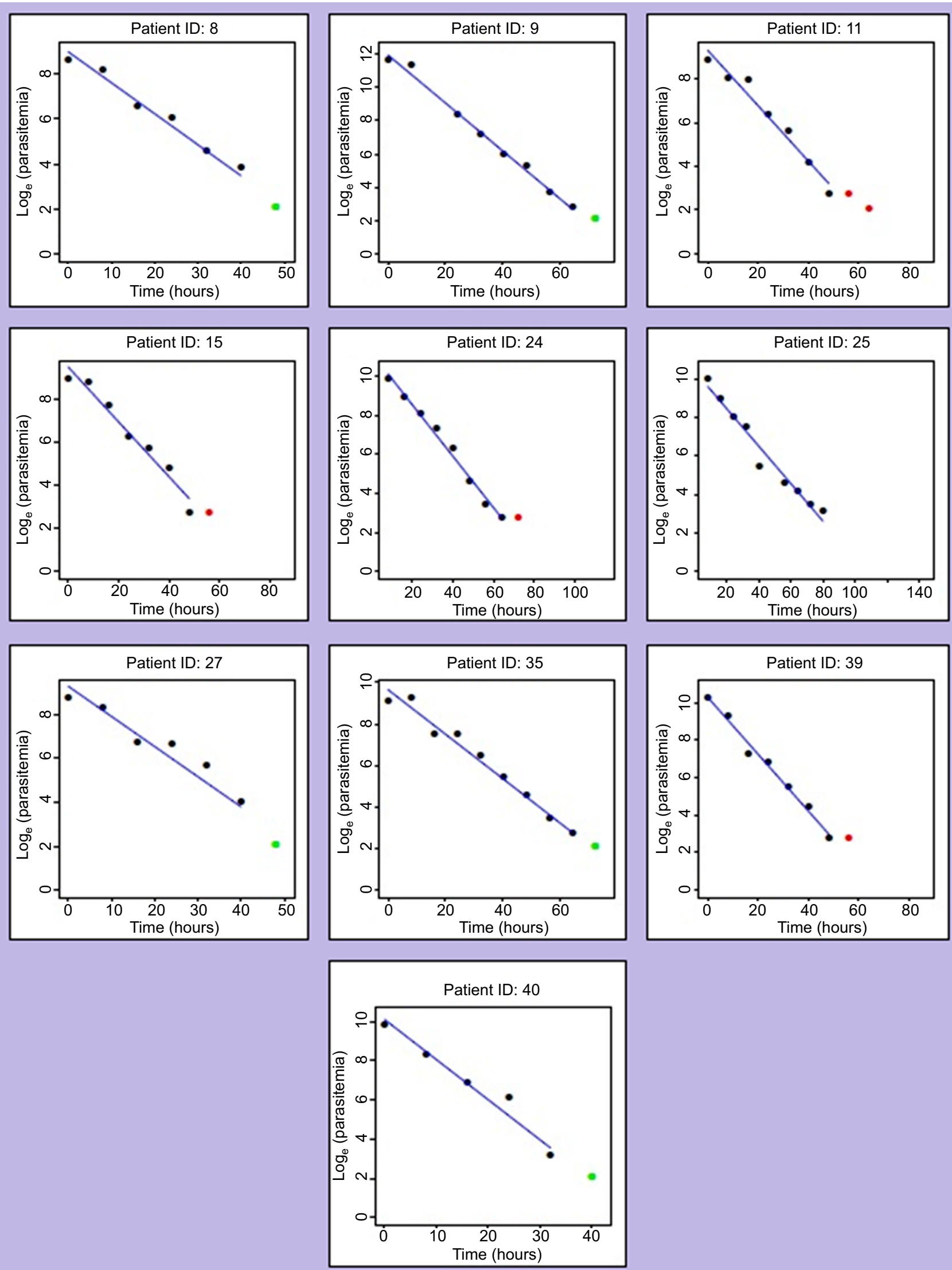

Figure I Parasite clearance curves $(n=10)$.

Notes: Black dots represent measurements used in the estimation of clearance rate. Red dots represent outliers excluded from calculation. Green dots represent measurements below the level of detection. 


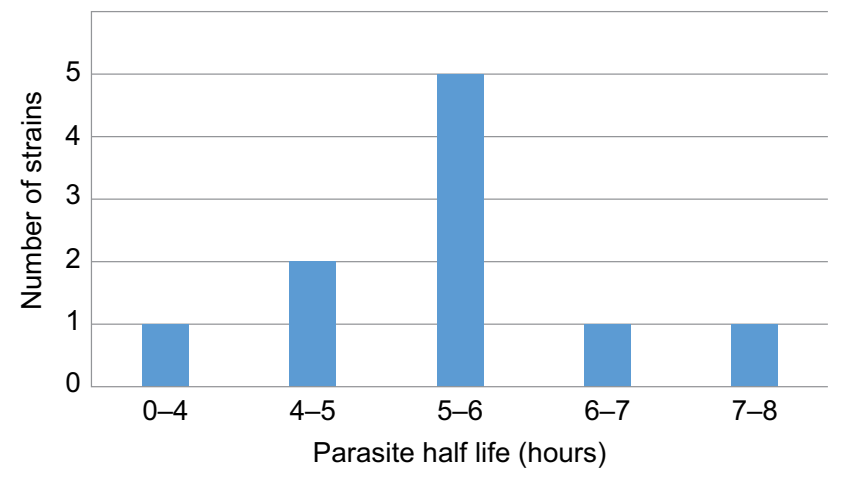

Figure 2 Distribution of parasite half-lives.

Table 2 Distribution slope half-life

\begin{tabular}{lll}
\hline Slope half-life (hours) & Total & Cumulative percentage \\
\hline $0-4$ & $\mathrm{I}$ & 10 \\
$4-5$ & 2 & 30 \\
$5-6$ & 5 & 80 \\
$6-7$ & 1 & 90 \\
$7-8$ & $\mathrm{I}$ & 100 \\
\hline
\end{tabular}

Note: Each interval includes the left-hand side value and not the right-hand side value.

Table 3 Summary of time to clear $50 \%, 90 \%, 95 \%$, and $99 \%$ of parasitemia (hours)

\begin{tabular}{llll}
\hline PC $^{\mathbf{a}}$ & Median & Range & IQR range \\
\hline PC50 & 8.86 & $4.49-15.16$ & $7.21-10.69$ \\
PC90 & 21.04 & $12.53-27.59$ & $18.45-25.70$ \\
PC95 & 26.29 & $15.92-34.73$ & $23.29-31.49$ \\
PC99 & 38.47 & $23.78-51.31$ & $34.52-43.85$ \\
\hline
\end{tabular}

Note: aParasite clearance time of, respectively, $50 \%, 90 \%, 95 \%$, and $99 \%$ of initial density.

Abbreviation: IQR, interquartile range.

to $2 \%$ after 3 days of artesunate followed by 3 days dihydroartemisinin-piperaquine in the same region. ${ }^{4}$

\section{Parasite clearance}

There was a lower observed positive parasitemia on day 2 $(62 \%)$ compared to that in the therapeutic efficacy study in 2011 (75\%). The day 3 parasitemia also showed a decrease (10\%) compared to the therapeutic efficacy trial in 2011, where a positive day 3 parasitemia of $31 \%$ was observed. ${ }^{9}$ In order to confirm the reduced sensitivity to artemisinin, as observed with artemether, this trial utilized artesunate monotherapy to exclude confounding effects of partner drugs. Artesunate and artemether are both absorbed rapidly and reach peak concentrations within 1-2 hours. Artesunate, expressed as dihydroartemisinin equivalents (the active antimalarial metabolite of artemisinin), reaches higher peak concentrations compared to artemether on day 1 when given in equal dihydroartemisinin dosages and even higher concentrations on day 2 compared to artemether resulting in a significant difference in the $C_{\max }$ value between artesunate and artemether $(8,088 \mathrm{nmol} / \mathrm{L}$ vs $3,454 \mathrm{nmol} / \mathrm{L}) .{ }^{14}$ This difference in pharmacokinetics resulting in higher peak concentrations with artesunate might account for the lower day 2 and day 3 parasitemia observed in this study.

The highest proportion of patients with a prolonged parasite clearance, found in western Cambodia and eastern Thailand, range up to $73 \%$, while these proportions still vary from $14 \%$ to $28 \%$ in northern Cambodia, Vietnam, and eastern Myanmar. ${ }^{4}$ The unimodal distribution of the parasite half-life $\sim 5-6$ hours was further in contrast with the bimodal distribution reported for southern Myanmar and may be the result of genetic homogeneity within the parasite population, prompting genetic analysis. ${ }^{13}$ Recently, Hastings et al stipulated that the use of the parasite half-life as a measure of drugefficacy may lead to overestimation of the drug effectiveness, as even quite substantial reductions in drug sensitivity may not be detected as slower clearance rates. Human immunity as the primary determinant of parasite clearance is further not included in the calculating mechanism. ${ }^{15}$ Therefore, although the limited number of analyzable data series in our study precludes clear conclusions about the parasite clearance rate and half-life in particular, the seven out of the ten (70\%) evaluated data series with a parasite half-life $\geq 5$ hours still comprise $17.9 \%(7 / 39)$ of the total data series and could thus still imply a possible emerging artemisinin resistance.

\section{Limitations of the study}

The occurrence of malaria predominantly in artisanal gold mining populations and the very low number of cases in our region once again emphasized the difficulty encountered in this susceptible group for enrollment of a sufficient number of patients and to obtain adequate follow-up rates. This obstacle denotes the difficulty present in obtaining adequate control of the disease.

Limitations of sample size were accentuated, due to compromised slide preservation, reducing the number of analyzable data series with nine data series. Furthermore, an adequate estimation of the different parasite clearance kinetics parameters with the WWARN parasite clearance estimator requires an initial parasitemia $\geq 4,000$ asexual parasites $/ \mu \mathrm{L}$, which accounted for a further reduction of 20 data series. Pharmacokinetic analysis was not done in this study.

\section{Conclusion}

As the lower day 3 parasitemia of $10 \%$ observed with artesunate monotherapy in our current study compared to the earlier therapeutic efficacy study in Suriname in 2011 can 
be ascribed to different pharmacokinetics between artesunate and artemether, the presence of at least $17.9 \%$ of patient data series with a parasite half-life $\geq 5$ hours supports the presence of delayed parasite clearance and emphasizes the earlier warning toward a possible emerging artemisinin resistance in Suriname as previously stated. ${ }^{9}$ The earlier reported increased copy number of $p f m d r l$ in Suriname, which has been linked with resistance to mefloquine and lumefantrine (partner drug in the current first-line ACT), further prompts vigilance for resistance to ACT in Suriname. ${ }^{16}$ Further studies are needed to examine artemisinin resistance in Suriname. The occurrence of malaria predominantly in artisanal gold mining populations will further govern the implementation measures for resistance containment.

\section{Acknowledgments}

We are grateful for the assistance of Truus Derks for her valued evaluation of microscopic slides and Doctor Mehul Dhorda with analysis of the data using the WWARN clearance estimator. This study received funding from the WHO, Geneva, Switzerland.

\section{Author contributions}

SGSV and MRA designed the study protocol. JKJ coordinated the study. SGSV supervised the study. JKJ and RDB analyzed and interpreted the data. SGSV, JKJ, RDB, and MRA drafted the article. SGSV, MRA, and RDB critically revised the article for intellectual content. SGSV is the guarantor of the article. All authors read and approved the final article.

\section{Disclosure}

The authors report no other conflicts of interest in this work.

\section{References}

1. Global Malaria Programme. Artemisinin and Artemisinin-Based Combination Therapy Resistance. World Health Organization; 2016. Available from: http://apps.who.int/iris/bitstream/10665/208820/1/ WHO_HTM_GMP_2016.5_eng.pdf. Accessed August 5, 2016.
2. Stepniewska K, Ashley E, Lee SJ, et al. In vivo parasitological measures of artemisinin susceptibility. $J$ Infect Dis. 2010;201:570-579.

3. Flegg J, Guerin P, White N, Stepniewska K. Standardizing the measurement of parasite clearance in falciparum malaria; the parasite clearance estimator. Malar J. 2011;10:339.

4. Ashley EA, Dhorda M, Fairhurst RM, et al. Spread of artemisinin resistance in Plasmodium falciparum malaria. N Engl J Med. 2014;371: 411-423.

5. Global Malaria Programme. Status ReportonArtemisinin Resistance. World Health Organization; 2014. Available from: http://www.who.int/malaria/ publications/atoz/status-rep-artemisinin-resistance-sep2014.pdf. Accessed April 27, 2015.

6. White NJ. The parasite clearance curve. Malar J. 2011;10:278.

7. WHO Global Malaria Programme. World Malaria Report 2015. World Health Organization; 2015. Available from: http://apps.who.int/iris/ bitstream/10665/200018/1/9789241565158_eng.pdf?ua=1 Accessed August 23, 2016.

8. Vreden SGS, Jitan JK, Eersel MGM, et al. Antimalarial Efficacy in Suriname 2001-2006: Implications of Evidence-Based Therapy Change. Amazon Malaria Initiative; 2011. Available from: http://www.usaidami.org/extras/LinksMedia_AMI.AnnualAchievReport_091911.pdf. Accessed October 22, 2016.

9. Vreden SGS, Jitan JK, Bansie RD, Adhin MR. Evidence of an increased incidence of day 3 parasitaemia in Suriname: an indicator of the emerging resistance of Plasmodium falciparum to artemether. Mem Inst Oswaldo Cruz. 2013;108:968-973.

10. General Bureau of Statistics Suriname [webpage on the Internet]. 8th General Population and Housing Census in Suriname. General Bureau of Statistics Suriname; 2013. Available from: http://www. statistics-suriname.org/index.php/statistieken/downloads/category/ 30-censusstatistieken-2012. Accessed November 28, 2015.

11. Heemskerk M. Kleinschalige goudwinning in Suriname - Een overzicht van sociaaleconomische, politieke en milieu-aspecten. Centre for Latin American Research and Documentation; 2009. Available from: http:// www.cedla.uva.nl/20_research/pdf/vDijck/suriname_project/Kleinschalige.pdf. Accessed May 22, 2015.

12. Thriemer K, Hong NV, Rosanas-Urgell A, et al. Delayed parasite clearance after treatment with dihydroartemisinin-piperaquine in Plasmodium falciparum malaria patients in Central Vietnam. Antimicrob Agents Chemother. 2014;58:7049-7055.

13. Kyaw MP, Nyunt MH, Chit K, et al. Reduced susceptibility of Plasmodium falciparum to artesunate in Southern Myanmar. PLoS One. 2013; 8(3):e57689. doi: 10.1371/journal.pone.0057689.

14. Suputtamongkol Y, Newton PN, Angus B, et al. A comparison of oral artesunate and artemether antimalarial bioactivities in acute falciparum malaria. Br J Clin Pharmacol. 2001;52:655-661.

15. Hastings I, Kay K, Hodel EM. How robust are malaria parasite clearance rates as indicators of drug effectiveness and resistance? Antimicrob Agents Chemother. 2015;59(10):6428-6436.

16. Labadie-Bracho M, Adhin MR. Increased pfmdr1 copy number in Plasmodium falciparum isolates from Suriname. Trop Med Int Health. 2013;18(7):796-799.
Infection and Drug Resistance

Publish your work in this journal

Infection and Drug Resistance is an international, peer-reviewed openaccess journal that focuses on the optimal treatment of infection (bacterial, fungal and viral) and the development and institution of preventive strategies to minimize the development and spread of resistance. The journal is specifically concerned with the epidemiology of antibiotic
Dovepress

resistance and the mechanisms of resistance development and diffusion in both hospitals and the community. The manuscript management system is completely online and includes a very quick and fair peerreview system, which is all easy to use. Visit http://www.dovepress.com/ testimonials.php to read real quotes from published authors. 